\title{
Momentum Average approximation for models with boson-modulated hopping: the role of closed loops in the dynamical generation of a finite quasiparticle mass
}

\author{
Mona Berciu ${ }^{1}$ and Holger Fehske ${ }^{2}$ \\ ${ }^{1}$ Department of Physics and Astronomy, University of British Columbia, Vancouver, BC V6T 1Z1, Canada \\ ${ }^{2}$ Institut für Physik, Ernst-Moritz-Arndt-Universität Greifswald, D-17487 Greifswald, Germany
}

(Dated: August 27, 2018)

\begin{abstract}
We generalize the momentum average (MA) approximation to study the properties of single polarons in models with boson affected hopping, where the fermion-boson scattering depends explicitly on both the fermion's and the boson's momentum. As a specific example, we investigate the Edwards fermion-boson model in both one- and two-dimensions. In one dimension, this allows us to compare our results with Exact Diagonalization results, to validate the accuracy of our approximation. The generalization to two-dimensional lattices allows us to calculate the polaron's quasiparticle weight and dispersion throughout the Brillouin zone, and to demonstrate the importance of Trugman loops in generating a finite effective mass even when the free fermion has an infinite mass.
\end{abstract}

PACS numbers: 72.10.-d,71.10.Fd,71.38.-k

\section{INTRODUCTION}

One of the most common problems in condensed matter physics is that of understanding the behavior of a particle coupled to bosons from its environment, for example an electron interacting with phonons, magnons, or orbitons of its host crystal $\underline{\underline{1}} \underline{\underline{6}}$ The particle becomes "dressed" by a cloud of bosonic excitations that accompany it. The resulting composite object, generally known as a polaron, can have properties significantly different from that of the bare particle $\underline{\underline{7}} \underline{\underline{\underline{9}}}$

The theoretical study of these properties is rather difficult away from the various asymptotic regimes where perturbation theory holds. Of course, a large variety of numerical techniques have been developed to deal with such problems,,$\underline{10} \underline{-16}$ and many interesting results have been uncovered, although the focus so far has been primarily on rather simple models such as the Holstein Hamiltonian $\frac{17,18}{18}$ that describes the simplest possible electronphonon coupling. The progress on analytical approximations that can efficiently yet accurately describe the nonperturbative regimes has been slower. In fact, it is only recently that the so-called Momentum Average (MA) approximation has been proposed for the Holstein model, and shown to accurately capture its polaronic behavior in all the parameter space except the extreme adiabatic limit $\underline{19} \mathrm{~A}$ way to systematically improve this approximation, as well as generalizations to certain kinds of more complex models have been proposed since $\underline{20}-26$ The availability of such simple yet accurate approximations is important, as it allows one to quickly explore large regions of the parameter space to identify the interesting properties of the model.

In this work we present the generalization of MA-type methods to calculate single polaron Green's functions for Hamiltonians whose hopping is boson affected. The bosons are assumed to be dispersionless, i.e. of Einstein type. For most polaron models, including the one discussed here, the spin of the fermion is irrelevant and we ignore it. Exceptions occur, for example, in systems with spin-orbit coupling, where suitable generalizations can be implemented. 24 The fermion moves on a $d$-dimensional lattice, which for simplicity is assumed to be hypercubic (generalization to other types of lattices is straightforward $\left.{ }^{23}\right)$. The cases $d=1$ and $d=2$ for the Edwards fermion-boson mode ${ }^{27}$ are discussed in detail and interesting physics related to the role of closed loops, possible in two dimensions (2D) but absent in 1D, is uncovered. We note that single polaron properties for this model have been investigated numerically in $1 \mathrm{D}, 28$ and we use these results to assess the accuracy of MA. We then extend our method to $2 \mathrm{D}$, where no results are currently available, and where we illustrate interesting effects of the boson modulated hopping. Other such models can be treated similarly.

The general form of the Hamiltonian of interest is:

$$
\begin{aligned}
\mathcal{H}= & \sum_{\mathbf{k}} \epsilon_{\mathbf{k}} c_{\mathbf{k}}^{\dagger} c_{\mathbf{k}}+\Omega \sum_{\mathbf{q}} b_{\mathbf{q}}^{\dagger} b_{\mathbf{q}} \\
& +\sum_{\mathbf{k}, \mathbf{q}} \frac{g(\mathbf{k}, \mathbf{q})}{\sqrt{N}} c_{\mathbf{k}-\mathbf{q}}^{\dagger} c_{\mathbf{k}}\left(b_{\mathbf{q}}^{\dagger}+b_{-\mathbf{q}}\right) .
\end{aligned}
$$

Here, $c_{\mathbf{k}}$ and $b_{\mathbf{q}}$ are fermion, respectively boson annihilation operators, and $N$ is the number of sites in the system. In all our results we assume periodic boundary conditions and let $N \rightarrow \infty$, however finite size systems and/or other types of boundary conditions can be treated similarly. The sums are over the Brillouin zone, $\epsilon_{\mathbf{k}}$ is the free-fermion dispersion while $\Omega$ is the bosons' energy (we set $\hbar=1$ ). Note that in (1) the fermion-boson scattering depends explicitly on both the fermion's and the boson's momentum. This is to be contrasted with simpler cases, such as the Holstein model, where $g(\mathbf{k}, \mathbf{q}) \rightarrow g$ is a constant, or models where the bosons modulate only on-site energies but not the hopping integrals, for which $g(\mathbf{k}, \mathbf{q}) \rightarrow g(\mathbf{q})$. The accuracy of MA approximations for these simpler types of Hamiltonians has been demonstrated in Refs. 1921. 
We are interested in calculating the single polaron Green's function, defined as

$$
G(\mathbf{k}, \omega)=\left\langle 0\left|c_{\mathbf{k}} \hat{G}(\omega) c_{\mathbf{k}}^{\dagger}\right| 0\right\rangle,
$$

where $|0\rangle$ is the vacuum, $\hat{G}(\omega)=[\omega-\mathcal{H}+i \eta]^{-1}$ is the resolvent associated with the Hamiltonian $\mathcal{H}$, and $\eta$ is a positive, infinitesimally small number.

From the Green's function we get the spectral weight

$$
A(\mathbf{k}, \omega)=-\frac{1}{\pi} \operatorname{Im} G(\mathbf{k}, \omega)
$$

which is measurable by (Inverse) Angle-Resolved Photoemission Spectroscopy 29 The lowest-energy pole of $A(\mathbf{k}, \omega)$ allows us to identify the polaron dispersion $E(\mathbf{k})$. Its residue is the quasiparticle $(q p)$ weight,

$$
Z_{\mathbf{k}}=\left|\left\langle\phi_{\mathbf{k}}\left|c_{\mathbf{k}}^{\dagger}\right| 0\right\rangle\right|^{2}
$$

i.e. the overlap between the polaron eigenfunction $\left|\phi_{\mathbf{k}}\right\rangle$, where $\mathcal{H}\left|\phi_{\mathbf{k}}\right\rangle=E(\mathbf{k})\left|\phi_{\mathbf{k}}\right\rangle$, and a free-fermion state. Of course, the spectral weight contains information about the higher energy states as well, but here we will primarily focus on the low-energy polaron band.

The article is organized as follows. We first introduce the Edwards fermion-boson model, which is the specific model with boson-modulated hopping that we will use as an example in this work. We then outline the MA approximation for the simpler 1D case, and use comparison with available numerical results to analyze its accuracy in various regimes. Then, we generalize MA for the $2 \mathrm{D}$ case and use it to understand the relevance of closed loops for generating a dynamical mass for the dresses quasiparticle. Finally we summarize our results and conclude.

\section{THE EDWARDS FERMION-BOSON MODEL}

The Edwards fermion-boson mode ${ }^{27,28}$ is defined by the Hamiltonian

$$
\mathcal{H}=-t_{f} \sum_{\langle i, j\rangle} c_{i}^{\dagger} c_{j}+\Omega \sum_{i} b_{i}^{\dagger} b_{i}-t_{b} \sum_{\langle i, j\rangle} c_{i}^{\dagger} c_{j}\left(b_{j}^{\dagger}+b_{i}\right)
$$

where the first term describes nearest-neighbor (NN) hopping of the fermion on the lattice of interest, the second term describes the Einstein boson branch, and the last term is the boson-modulated hopping. Note that in the limit $t_{f} \rightarrow 0$, it is only the last term that allows the fermion to move: hopping from one site to a neighboring one either creates an excitation at the "departure" site, or removes one from the "arrival" site. This model provides a way to mimic, for example, the motion of a fermion through an antiferromagnetically ordered spin background ${ }^{3,30,33}$ For a Neél antiferromagnet (AFM) doped with one fermion-which is the zero-order description of a hole moving in a cuprate $\mathrm{CuO}_{2}$ layerthe hopping of the fermion reshuffles the spins along its

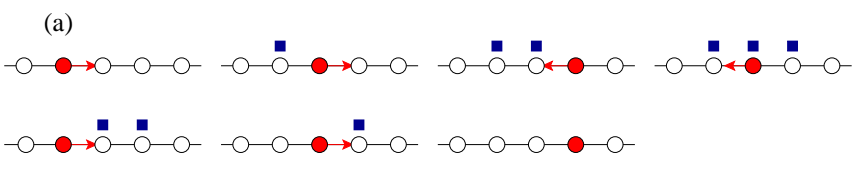

(b)
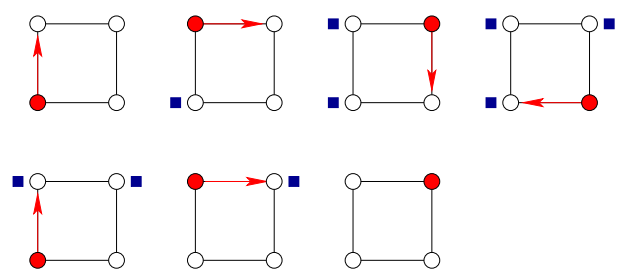

FIG. 1. Sketch of the 3-boson, 3-site sequence of processes that give rise to effective second $\mathrm{NN}$ fermion hopping. The site occupied by the fermion is shaded and the arrow indicates the direction of the next $t_{b}$-hopping process. Each $t_{b}$-hopping either leaves a boson (drawn as a square) at the initial site, or absorbs a boson from the arrival site. Both collinear (a) and closed loop (b) processes are allowed in $2 \mathrm{D}$; in $1 \mathrm{D}$ only (a) is possible.

path. If the spin at the "arrival" site has the proper orientation, when it is shuffled to the starting site as the fermion hops it will have the wrong orientation (a "magnon" defect is created at the initial site). Vice versa, if the visited spin has the wrong orientation, when shuffled by one site it will be properly aligned ("magnon" defect removed from the arrival site). This is precisely the type of physics described by this boson-modulated hopping, although it ignores details such as the hardcore boson constraint for magnons, the fact that in a Neél AFM the energy of neighboring magnons is not additive, and also it allows the particle to coexist with bosons at the same site. The free-fermion hopping term $t_{f}$, on the other hand, has to be added when describing motion through a Heisenberg-like AFM, where spin fluctuations continuously create and annihilate magnons. Indeed, as shown in Refs. 28 and 34, the free-fermion hopping term can be mapped into a purely bosonic term of the type $\lambda \sum_{i}\left(b_{i}+b_{i}^{\dagger}\right)$ with $\lambda=t_{f} \Omega /\left(2 t_{b}\right)$, which allows the number of magnons to fluctuate.

The "conventional wisdom" is that a fermion in a $2 \mathrm{D}$ Neél AFM $\left(t_{f}=0\right.$ case $)$ has an infinite effective mass, because as it tries to move it creates a costly string of defects which effectively pin it to its original site. This is, however, not true. The boson-modulated hopping gives rise to an effective fermion mass even when the bare fermionic mass is infinite $\left(t_{f}=0\right) \stackrel{28,33}{=}$ For the $1 \mathrm{D}$ chain, this is primarily due to the 3 -site, 3 -boson processes sketched in Fig. 1(a) which result in an effective second NN hopping of the fermion (of course, more complicated processes involving more bosons are also possible, but they are energetically more costly). In Ref. 30 , it was noted that in $2 \mathrm{D}$, these collinear processeswhich in $2 \mathrm{D}$ give rise to effective $3 \mathrm{rd} \mathrm{NN}$ hopping - are supplemented by the closed loop processes sketched in 
Fig. 1(b), which give rise to effective 2nd NN hopping. The importance of such closed loops - known as Trugman paths - for determining the effective quasiparticle mass has been emphasized already in Ref. 30 in the context of cuprates. In this work, we are the first to explicitly investigate this phenomenology for the 2D Edwards fermion-boson model.

While we focus on this Hamiltonian for the remainder of this work, the MA method can be generalized straightforwardly to other boson-modulated hoppings, like that appearing in the 1D Su-Schrieffer-Heeger model of polyacetylene $\stackrel{35}{\underline{3}}$ Its phonon-modulated hopping term is proportional to $\sum_{i}\left(c_{i}^{\dagger} c_{i+1}+\right.$ H.c. $)\left[b_{i}^{\dagger}+b_{i}-b_{i+1}^{\dagger}-b_{i+1}\right]$, i.e. here phonons can be both absorbed and created at either of the two sites involved in the hopping process.

\section{THE MOMENTUM AVERAGE APPROXIMATION}

One can discuss the meaning of the MA approximations from several different points of view. One is that this is an approximation which sums semi-analytically all diagrams contributing to the self-energy, however "exponentially small" contributions are ignored when calculating the expression of each such diagram. $\frac{20}{2}$ The precise meaning of this statement will be clarified below.

A more useful starting point for our purposes here is the variational meaning of MA 20,31 The central idea of polaron physics is that the dressed quasiparticle - the polaron - consists of the fermion itself and a cloud of bosons in its vicinity. MA permits one to systematically select what bosonic states to keep in the variational space to describe this cloud, and sum their contributions efficiently. Of course, the more states kept, the more accurate the results. Physical intuition is needed to decide what is a minimal acceptable starting point.

In this section, we first describe the MA approximation for a $1 \mathrm{D}$ chain, and assess its accuracy against available numerical data. We then briefly review the $2 \mathrm{D}$ generalization and analyze the resulting physics, which has not been investigated before.

\section{A. MA for the 1D chain}

Since we primarily focus on the low-energy polaronic physics, we will only attempt to describe the polaronic cloud. The only restriction we impose regards its spatial size, i.e., what is the maximum number of neighboring sites that it can span. The number of bosons at any site in the cloud, as well as the position of the fermion with respect to the center of the cloud, are not restrictedthey can take any values. A few of the possible states for a 3-site cloud calculation are sketched in Fig. 2(a). Of course, any 1- and 2-site cloud states belongs to this set.

Note that here we do not allow any bosonic excitations to occur far from the polaron cloud, since they primarily (a)

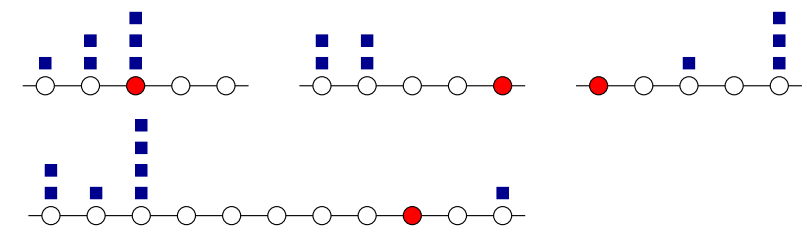

FIG. 2. (Color online) (a) Sketch of several states included in the $\mathrm{MA}^{(0)}$ approximation discussed here. The polaron cloud is allowed to extend on up to three neighboring sites, located anywhere in the system with any number of bosons (shown as red squares) at each site, and the fermion (shown as a shaded blue circle) at any distance away from the cloud. (b) Example of extra states included in a $\mathrm{MA}^{(1)}$ level approximation, with a boson arbitrarily far away from the polaron cloud.

contribute to higher-energy states. In other words, this will be a $\mathrm{MA}^{(0)}$ level approximation 20 Generalization to $\mathrm{MA}^{(1)}$ and higher levels, needed for example to describe the polaron+one-boson continuum, is straightforward although fairly cumbersome. For example, in $\mathrm{MA}^{(1)}$ we also include states such as sketched in Fig. 2(b), with one boson arbitrarily far away from the polaron cloud. Similarly $\mathrm{MA}^{(2)}$ allows two bosons at arbitrary locations away from the cloud, etc. As detailed below, the physics encoded in these higher-level approximations does not lead to any qualitative changes for the properties and parameter ranges that we are interested in.

After deciding on the $\mathrm{MA}^{(0)}$ (henceforth called simply MA) level, the only question left is how big should one allow the polaron cloud to be. For the Holstein model, a one-site cloud already gives a remarkably accurate description in any dimension, most everywhere in the parameter space except intermediate coupling in the adiabatic limit $\Omega / t \rightarrow 0, \underline{\underline{19,20}}$

For the Edwards model, as already discussed, at least 3 -site boson clouds need be considered in order to describe the leading processes that result in the dynamical generation of a finite effective mass in the limit $t_{f}, \lambda \rightarrow 0$. As a result, we will start directly by building a variational MA approximation allowing any number of bosons on any 3 consecutive sites, which can be located at any distance from where the fermion is. To achieve this, we introduce 3 types of generalized Green's functions. The first are

$$
F_{n}(k, q, \omega)=\sum_{i} e^{i(k-q) R_{i}}\left\langle 0\left|c_{k} \hat{G}(\omega) c_{q}^{\dagger} b_{i}^{\dagger^{n}}\right| 0\right\rangle,
$$

where the ket describes a state of total momentum $k$ (as required since the Hamiltonian is invariant to translations) of which the fermion has a momentum $q$ and the cloud of $n$ bosons, all located at the same site, has momentum $k-q$.

Next are the two-site cloud Green's functions, namely

$$
F_{n, m}(k, q, \omega)=\sum_{i} e^{i(k-q) R_{i}}\left\langle 0\left|c_{k} \hat{G}(\omega) c_{q}^{\dagger} b_{i}^{\dagger^{m}}{b^{\dagger}}_{i+1}^{n-m}\right| 0\right\rangle
$$


which are defined only for $n \geq 2$ and $1 \leq m \leq n-1$, so that they are distinct from the one-site cloud functions
$F_{n}(k, q, \omega)$ defined above. Finally, we have the three-site cloud functions,

$$
F_{n, m, p}(k, q, \omega)=\sum_{i} e^{i(k-q) R_{i}}\left\langle 0\left|c_{k} \hat{G}(\omega) c_{q}^{\dagger}{b^{\dagger}}_{i-1}^{m}{b^{\dagger}}_{i}^{n-m-p} b^{\dagger}{ }_{i+1}^{p}\right| 0\right\rangle
$$

defined for $n \geq 2$ and $1 \leq m \leq n-1,1 \leq p \leq n-1, m+$ $p \leq n$. These restrictions again avoid overlap with the functions introduced above. Note, however, that they allow bosons to exist only on the two outer sites of the three-site cloud when $m+p=n$; such states are not accounted for by $F_{n, m}(k, q, \omega)$. In principle one can keep adding other states, either with more extended clouds, or with bosons far away from the cloud, until convergence is achieved. For reasons already explained, for this model we expect that it suffices to stop here.

The next step is to generate equations of motion for these generalized Green's functions. These are obtained by using the Dyson identity $\hat{G}(\omega)=\hat{G}_{0}(\omega)+$ $\hat{G}(\omega) \mathcal{V} \hat{G}_{0}(\omega)$, where $\hat{G}_{0}(\omega)$ is the resolvent for $\mathcal{H}_{0}=$ $\mathcal{H}-\mathcal{V}$. This is an exact (non-perturbative) identity and holds for any partitioning of $\mathcal{H}=\mathcal{H}_{0}+\mathcal{V}$. For our purposes, it is convenient to take $\mathcal{H}_{0}$ as the non-interacting part and $\mathcal{V}$ as the boson-modulated hopping term. Note that the kets at the right of $\hat{G}(\omega)$ in all the above definitions are eigenstates of $\mathcal{H}_{0}$, so that, for e.g.

$$
\hat{G}_{0}(\omega) c_{q}^{\dagger} b_{i}^{\dagger}{b^{\dagger}}_{i+1}^{n-m}|0\rangle=G_{0}(q, \omega-n \Omega) c_{q}^{\dagger} b_{i}^{\dagger}{b^{\dagger}}_{i+1}^{n-m}|0\rangle,
$$

where

$$
G_{0}(k, \omega)=\frac{1}{\omega+i \eta-\epsilon_{k}}
$$

is the free fermion propagator. With this observation, we find the exact equation of motion for $G(k, \omega)$ to be

$$
G(k, \omega)=G_{0}(k, \omega)\left[1-t_{b} \sum_{i} \frac{e^{i k R_{i}}}{\sqrt{N}}\left\langle 0\left|c_{k} \hat{G}(\omega)\left(c_{i-1}^{\dagger}+c_{i+1}^{\dagger}\right) b_{i}^{\dagger}\right| 0\right\rangle\right]
$$

because $\mathcal{V} c_{i}^{\dagger}|0\rangle=-t_{b}\left(c_{i-1}^{\dagger}+c_{i+1}^{\dagger}\right) b_{i}^{\dagger}|0\rangle$. This shows that the Green's function of interest to us is linked to various averages over the Brillouin zone (momentum averages) of the $F_{1}(k, q, \omega)$ Green's functions. To make this more precise, we introduce the real-space counterparts of the generalized Green's functions defined above, namely:

$$
\begin{gathered}
f_{n}(k, \delta, \omega)=\frac{1}{N} \sum_{q} e^{i q \delta a} F_{n}(k, q, \omega)=\sum_{i} \frac{e^{i k R_{i}}}{\sqrt{N}}\left\langle 0\left|c_{k} \hat{G}(\omega) c_{i-\delta}^{\dagger} b_{i}^{\dagger}{ }_{i}^{n}\right| 0\right\rangle, \\
f_{n, m}(k, \delta, \omega)=\frac{1}{N} \sum_{q} e^{i q \delta a} F_{n, m}(k, q, \omega)=\sum_{i} \frac{e^{i k R_{i}}}{\sqrt{N}}\left\langle 0\left|c_{k} \hat{G}(\omega) c_{i-\delta}^{\dagger} b_{i}^{\dagger^{m}} b^{\dagger}{ }_{i+1}^{n-m}\right| 0\right\rangle,
\end{gathered}
$$

and

$$
f_{n, m, p}(k, \delta, \omega)=\frac{1}{N} \sum_{q} e^{i q \delta a} F_{n, m, p}(k, q, \omega)=\sum_{i} \frac{e^{i k R_{i}}}{\sqrt{N}}\left\langle 0\left|c_{k} \hat{G}(\omega) c_{i-\delta}^{\dagger} b_{i-1}^{\dagger}{ }_{i}^{m} b_{i}^{\dagger^{n-m}-p} b_{i+1}^{\dagger}{ }_{i+1}\right| 0\right\rangle
$$

The kets on the right of the resolvent continue to describe a state of total momentum $k$, however with the fermion fixed at a certain distance $\delta a$ away from the boson cloud. Here $a$ is the lattice distance, so that $\delta$ are integers. These definitions do not necessarily lead to the most "esthetic" final equations, but they are handy and generalize easily to higher dimensions. More symmetric 1D equations can be found if we use the sin and cos, instead of complex
Fourier transforms.

With these notations, we have the exact equation

$$
G(k, \omega)=G_{0}(k, \omega)\left[1-t_{b}\left(f_{1}(k, 1, \omega)+f_{1}(k,-1, \omega)\right)\right] .
$$

Let us consider now the equation of motion for $F_{n}(k, q, \omega)$ with $n \geq 1$. When acting on $c_{q}^{\dagger} b_{i}^{\dagger}|0\rangle$, both the boson annihilation and the boson creation part of $\mathcal{V}$ will contribute. The boson annihilation contribution can 
be calculated exactly, since bosons can only be annihilated at the one site where they are present. However, the boson creation part can add a boson either at the site where the cloud is, or to any other site because a fermion in the state $c_{q}^{\dagger}|0\rangle$ is delocalized over the entire chain. Of course, we do not expect all these outcomes to be equally likely, and it is this that allows us to make progress.
This is where the MA approximation is made: we only allow new bosons to be created within at most two-site distance from where the one-site boson cloud is. In other words, we allow the boson cloud to extend spatially but not over more than 3 consecutive sites, since we decided that this is our variational space. After all possible such terms are accounted for, we find that within this MA approximation:

$$
\begin{aligned}
& F_{n}(k, q, \omega)=-t_{b} G_{0}(q, \omega-n \Omega)\left[\left(e^{i q a}+e^{-i q a}\right) n f_{n-1}(k, 0, \omega)+\left[f_{n+1}(k, 1, \omega)+f_{n+1}(k,-1, \omega)\right]\right. \\
& \quad+e^{-i q a+i k a}\left[f_{n+1,1}(k, 1, \omega)+f_{n+1,1}(k,-1, \omega)\right]+e^{i q a}\left[f_{n+1, n}(k, 0, \omega)+f_{n+1, n}(k,-2, \omega)\right] \\
& \left.\quad+e^{-2 i q a+i k a}\left[f_{n+1,1, n}(k, 2, \omega)+f_{n+1,1, n}(k, 0, \omega)\right]+e^{2 i q a-i k a}\left[f_{n+1, n, 1}(k, 0, \omega)+f_{n+1, n, 1}(k,-2, \omega)\right]\right] .
\end{aligned}
$$

The terms on the first line come from the exact boson annihilation part (the first) and the boson creation term where the new boson is added at the site where the cloud is (the last). The terms in the second line describe the contributions where the new boson is created on a $\mathrm{NN}$ site of the cloud. The terms on the 3rd line are the contributions when the new boson is created on a second NN site of the cloud.

Within the same approximation, we also find

$$
\begin{aligned}
& F_{n, m}(k, q, \omega)=-t_{b} G_{0}(q, \omega-n \Omega)\left[\left(e^{i q a}+e^{-i q a}\right) m f_{n-1, m-1}(k, 0, \omega)+\left(1+e^{2 i q a}\right)(n-m) f_{n-1, m}(k,-1, \omega)\right. \\
& \quad+e^{i q a}\left[f_{n+1, m}(k, 0, \omega)+f_{n+1, m}(k,-2, \omega)\right]+\left[f_{n+1, m+1}(k, 1, \omega)+f_{n+1, m+1}(k,-1, \omega)\right] \\
& \left.\quad+e^{2 i q a-i k a}\left[f_{n+1, m, 1}(k, 0, \omega)+f_{n+1, m, 1}(k,-2, \omega)\right]+e^{-i q a}\left[f_{n+1,1, n-m}(k, 0, \omega)+f_{n+1,1, n-m}(k, 2, \omega)\right]\right]
\end{aligned}
$$

and

$$
\begin{aligned}
F_{n, m, p} & (k, q, \omega)=-t_{b} G_{0}(q, \omega-n \Omega)\left[\left(e^{-2 i q a}+1\right) m f_{n-1, m-1, p}(k, 1, \omega)+\left(e^{i q a}+e^{-i q a}\right)(n-m-p) f_{n-1, m, p}(k, 0, \omega)\right. \\
& +\left(1+e^{2 i q a}\right) p f_{n-1, m, p-1}(k,-1, \omega)+e^{-i q a}\left[f_{n+1, m+1, p}(k, 0, \omega)+f_{n+1, m+1, p}(k, 2, \omega)\right] \\
& \left.+\left[f_{n+1, m, p}(k, 1, \omega)+f_{n+1, m, p}(k,-1, \omega)\right]+e^{i q a}\left[f_{n+1, m, p+1}(k, 0, \omega)+f_{n+1, m, p+1}(k,-2, \omega)\right]\right] .
\end{aligned}
$$

As before, creation processes are only allowed to add extra bosons so that the total cloud does not extend over more than 3 consecutive sites. The annihilation processes are treated exactly, however one has to be careful when there is a single boson on an outside site of the cloud. If this is the annihilated boson, the size of the cloud decreases. As a result:

$$
\begin{aligned}
& f_{n-1,0}(k, \delta, \omega) \rightarrow e^{-i k a} f_{n-1}(k, \delta+1, \omega), \\
& f_{n-1, n-1}(k, \delta, \omega) \rightarrow f_{n-1}(k, \delta, \omega), \\
& f_{n-1,0, p}(k, \delta, \omega) \rightarrow f_{n-1, n-1-p}(k, \delta, \omega), \text { if } p<n-1 \\
& f_{n-1,0, n-1}(k, \delta, \omega) \rightarrow e^{-i k a} f_{n-1}(k, \delta+1, \omega), \\
& f_{n-1, m, 0}(k, \delta, \omega) \rightarrow e^{i k a} f_{n-1, m}(k, \delta-1, \omega), \text { if } m<n-1, \\
& f_{n-1, n-1,0}(k, \delta, \omega) \rightarrow e^{i k a} f_{n-1}(k, \delta-1, \omega) .
\end{aligned}
$$

All these identities follow directly from the definitions of Eqs. (11)-(13).

We have thus generated an infinite system of coupled equations of motion linking various Green's functions with a total of $n$ bosons to Green's functions with $n-1$ and $n+1$ bosons. The only approximation is the restriction on the size of the allowed boson cloud. A solution of this system will give $G(k, \omega)$ within this MA approximation, together with all the other generalized Green's functions from which we can extract additional information on the structure of the polaron cloud. ${ }^{32}$

This solution is straightforward to obtain in terms of the momentum averaged Green's functions $f_{n}$. First, note that only a finite number of these are needed, for a given value of $n$, in order to be able to calculate everything else. For example, only $f_{n, m, p}(k, \delta, \omega)$ with $|\delta| \leq 2$ appear on the right hand side of all these equations, and similar bounds can be found for the other functions. We 


$$
\begin{aligned}
& f_{n}(\delta)=-t_{b}\left[g_{0}\left(\delta+1, \omega_{n}\right)+g_{0}\left(\delta-1, \omega_{n}\right)\right] n f_{n-1}(0)-t_{b} g_{0}\left(\delta, \omega_{n}\right)\left[f_{n+1}(1)+f_{n+1}(-1)\right] \\
& \quad-t_{b} g_{0}\left(\delta-1, \omega_{n}\right) e^{i k a}\left[f_{n+1,1}(1)+f_{n+1,1}(-1)\right]-t_{b} g_{0}\left(\delta+1, \omega_{n}\right)\left[f_{n+1, n}(0)+f_{n+1, n}(-2)\right] \\
& \quad-t_{b} g_{0}\left(\delta-2, \omega_{n}\right) e^{+i k a}\left[f_{n+1,1, n}(2)+f_{n+1,1, n}(0)\right]-t_{b} g_{0}\left(\delta+2, \omega_{n}\right) e^{-i k a}\left[f_{n+1, n, 1}(0)+f_{n+1, n, 1}(-2)\right],
\end{aligned}
$$

$$
\begin{aligned}
& f_{n, m}(\delta)=-t_{b}\left[g_{0}\left(\delta+1, \omega_{n}\right)+g_{0}\left(\delta-1, \omega_{n}\right)\right] m f_{n-1, m-1}(0)-t_{b}\left[g_{0}\left(\delta, \omega_{n}\right)+g_{0}\left(\delta+2, \omega_{n}\right)\right](n-m) f_{n-1, m}(-1) \\
& \quad-t_{b} g_{0}\left(\delta+1, \omega_{n}\right)\left[f_{n+1, m}(0)+f_{n+1, m}(-2)\right]-t_{b} g_{0}\left(\delta, \omega_{n}\right)\left[f_{n+1, m+1}(1)+f_{n+1, m+1}(-1)\right] \\
& \quad-t_{b} g_{0}\left(\delta+2, \omega_{n}\right) e^{-i k a}\left[f_{n+1, m, 1}(0)+f_{n+1,1, n}(-2)\right]-t_{b} g_{0}\left(\delta-1, \omega_{n}\right)\left[f_{n+1,1, n-m}(0)+f_{n+1,1, n-m}(2)\right],
\end{aligned}
$$

and

$$
\begin{aligned}
& f_{n, m, p}(\delta)=-t_{b}\left[g_{0}\left(\delta-2, \omega_{n}\right)+g_{0}\left(\delta, \omega_{n}\right)\right] m f_{n-1, m-1, p}(1)-t_{b}\left[g_{0}\left(\delta-1, \omega_{n}\right)+g_{0}\left(\delta+1, \omega_{n}\right)\right](n-m-p) f_{n-1, m, p}(0) \\
& \quad-t_{b}\left[g_{0}\left(\delta, \omega_{n}\right)+g_{0}\left(\delta+2, \omega_{n}\right)\right] p f_{n-1, m, p-1}(-1)-t_{b} g_{0}\left(\delta-1, \omega_{n}\right)\left[f_{n+1, m+1, p}(0)+f_{n+1, m+1, p}(2)\right] \\
& \quad-t_{b} g_{0}\left(\delta, \omega_{n}\right)\left[f_{n+1, m, p}(1)+f_{n+1, m, p}(-1)\right]-t_{b} g_{0}\left(\delta+1, \omega_{n}\right)\left[f_{n+1, m, p+1}(0)+f_{n+1, m, p+1}(-2)\right]
\end{aligned}
$$

Here we used the shorthand notations $f_{n}(\delta) \equiv f_{n}(k, \delta, \omega)$ etc. and $\omega_{n} \equiv \omega-n \Omega$ in order to simplify the notation. Also,

$$
g_{0}(\delta, \omega)=\frac{1}{N} \sum_{k} e^{i k \delta a} G_{0}(k, \omega)
$$

are the free propagators in real space, which can be calculated analytically, 19,36 For any given number $n$ of bosons, the needed Green's functions are $f_{n, m, p}(k, \delta, \omega)$ with $|\delta| \leq 2, f_{n, m}(k, \delta, \omega)$ with $|\delta| \leq 3$ and $f_{n}(k, \delta, \omega)$ with $-3 \leq \delta \leq 4$. Once we know these, we can calculate all other $f$ and $F$ generalized Green's functions.

To solve this set of recurrence equations, we order all Green's functions with a given $n$ in a vector $V_{n}$ of dimension $7 n+1+5 n(n-1) / 2$. Then, for any $n \geq 1$, Eqs. (14)-(26) map into the matrix recurrence equations

$$
V_{n}=\alpha_{n}(k, \omega) V_{n-1}+\beta_{n}(k, \omega) V_{n+1},
$$

where $\alpha_{n}(k, \omega)$ and $\beta_{n}(k, \omega)$ are sparse matrices which can be read directly from Eqs. (14)-(26). The solution, for any $n \geq 1$, has the general form 19,20

$$
V_{n}=A_{n}(k, \omega) V_{n-1},
$$

where $A_{n}(k, \omega)$ are given by the continued fractions

$$
A_{n}(k, \omega)=\frac{\alpha_{n}(k, \omega)}{1-\beta_{n}(k, \omega) A_{n+1}(k, \omega)}
$$

starting from a large value $N$ with $A_{N}(k, \omega)=0$. The physical motivation for this choice has been discussed at

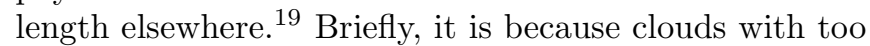
many bosons $N \rightarrow \infty$ are too expensive energetically, and therefore very unlikely to be observed. Hence, the propagators into such states must become vanishingly small for a large enough $N$. In practice one increases $N$ until the matrices $A_{n}(k, \omega)$ are converged to within any accuracy one chooses.

Consider now Eq. (29) for $n=1$. The entries in $V_{0}$ are the various $f_{0}(k, \delta, \omega)=e^{i k \delta a} G(k, \omega)$ (see Eq. (11); the functions $f_{n, m}$ and $f_{n, m, p}$ are defined only for $n \geq 2$ ). As a result, once we know the matrix $A_{1}(k, \omega)$, we find

$$
f_{1}(k, \pm 1, \omega)=a^{ \pm}(k, \omega) G(k, \omega),
$$

where $a^{ \pm}(k, \omega)$ are combinations of the appropriate matrix elements of $A_{1}(k, \omega)$ and $e^{i k \delta a}$ factors. Using this in Eq. (14) gives us the standard solution

$$
G(k, \omega)=\frac{1}{\omega+i \eta-\epsilon_{k}-\Sigma(k, \omega)}
$$

with a self-energy, at this level of MA approximation, of

$$
\Sigma(k, \omega)=-t_{b}\left[a^{+}(k, \omega)+a^{-}(k, \omega)\right] .
$$

In some limiting cases the model has certain symmetries that will insure that many of the generalized Green's functions vanish identically. A simple example is the case $t_{f}=0.3^{33}$ Then the free fermion dispersion vanishes, $\epsilon_{k}=-2 t_{f} \cos (k a) \rightarrow 0$, and so:

$$
g_{0}(\delta, \omega) \rightarrow \delta_{\delta, 0} \frac{1}{\omega+i \eta} .
$$

This simplifies Eqs. (14)-(26) significantly and one can show that many of the $f$ functions are identically zero. For example, only states $f_{2 n, n}$ and $f_{2 n+1, n}, f_{2 n+1, n+1}$ survive. This becomes obvious if one considers what kinds 
of boson clouds can be formed if one acts with $\mathcal{V}$ repeatedly on any one-fermion state. By continuity, one expects that for small $t_{f}$, these functions will still be very small although not precisely zero. This fact could be used to speed up the calculation in this limit, by removing these kets from the variational set and thus decreasing the size of all these matrices.

In fact, it is known that $g_{0}(\delta, \omega)$ decreases exponentially with increasing distance $|\delta|$ for values $\omega<$ $-2 d t_{f}$, i.e. below the free fermion continuum ( $d$ is the dimension) $\stackrel{36}{=}$ If we are interested in low-energy properties at $\omega<-2 d t_{f}$, and especially since $\omega_{n}=\omega-n \Omega$ appears in Eqs. (14)-(26), we see that most of the terms on the right are multiplied by exponentially small functions if $|\delta|>0$ in their corresponding $g_{0}\left(\delta, \omega_{n}\right)$ factor. This explains the earlier statement that the approximation ignores only exponentially small contributions. One can check that going to more extended clouds will bring in more terms in Eqs. (14)-(26), but their $g_{0}\left(\delta, \omega_{n}\right)$ factors will be even smaller. This argument only becomes problematic when the ground-state energy is not too far below the free-fermion continuum and $\Omega / t_{f} \rightarrow 0$. In this case, $g_{0}\left(\delta, \omega_{n}\right)$ still decays exponentially with increasing $\delta$ but very slowly, and much more extended clouds may form with significant probability. For the Holstein model, this leads to quantitative discrepancies in the MA prediction for intermediate couplings, where the polaron cloud can extend over many sites $\frac{37,38}{2}$ At strong couplings, the Lang-Firsov approach ${ }^{39}$ gives a small, one-site cloud polaron, and MA with a one-site cloud restriction becomes asymptotically exact. No exact asymptotic solution is known for the Edwards model, therefore there is no guarantee that in the limit $\Omega / t_{f} \rightarrow 0$, a 3 -site cloud will provide a good description. However, as we show below, MA allows us to decide, with a high level of confidence, whether it provides a reasonable description.

Finally, let us comment on why we chose to allow a three-site cloud, as oppose to a one-site or two-site one. Consider what would happen if we restricted the variational space to single-site clouds only. Then, we can set all functions $f_{n, m}$ and $f_{n, m, p}$ identically to zero. From Eq. (14), we see that the recurrence relation that now links together only the $f_{n}(\delta)$ with $\delta=-1,0,1$ does not contain any dependence of $k$ in any of its terms, resulting in a self-energy that is independent of $k$. For example, for $t_{f}=0$ this would mean that the polaron is also dispersionless. We know that this is not the case due to

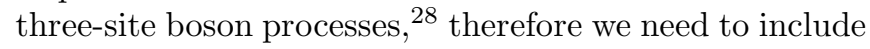
at least such cloud structures in the calculation to capture this. If $\Omega$ is not too small, one can argue that much more extended clouds are unlikely for energetical reasons: bosons far from the particle cost energy $\Omega$ to create yet are unlikely to interact with the particle because of the separation. Of course, one can increase the size of the cloud systematically until convergence is achieved. Such an exercise allows one to uncover the physics essential for explaining the properties of the polaron, from the importance of various terms play.

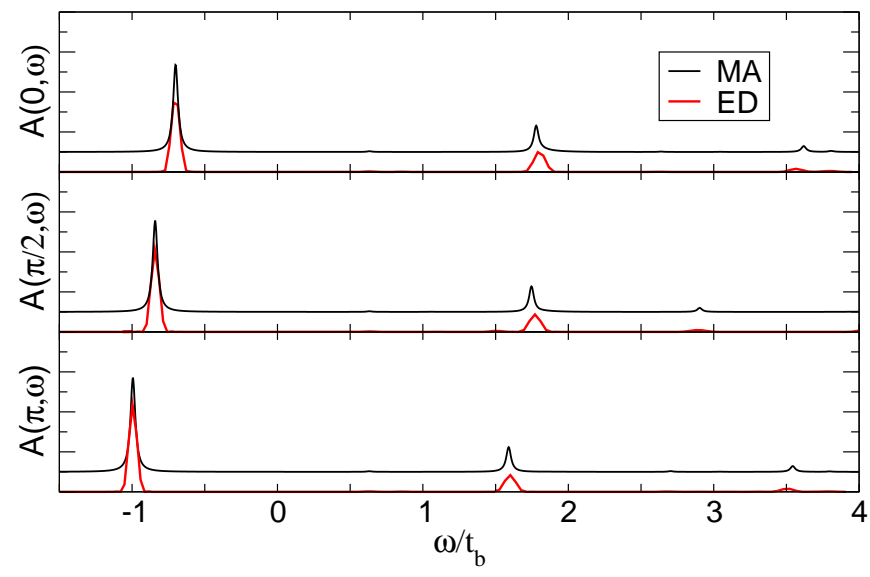

FIG. 3. (Color online) Spectral weights $A(k, \omega)$ for $k=0$ (top), $k=\frac{\pi}{2}$ (middle) and $k=\pi$ (bottom) from MA (black thin lines) and ED (red thick lines). The MA results are shifted upwards to ease the comparison. Parameters are $\Omega / t_{b}=2, t_{f} / t_{b}=0.1, \eta / t_{b}=0.02$.
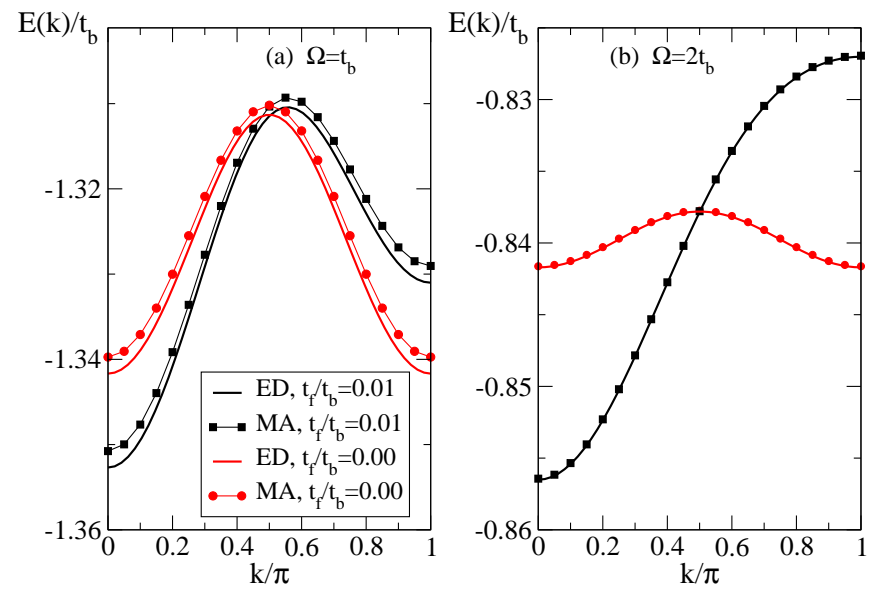

FIG. 4. (Color online) Polaron energy $E(k)$ in units of $t_{b}$ vs. $k$, for the parameters indicated. Lines show ED results, symbols show MA results (dashed lines are guides to the eye).

\section{B. MA results for the 1D Edwards model}

We can analyze the accuracy of the MA approximation for the Edwards model in 1D, where accurate numerical results have been obtained using variational Exact Diagonalization (ED) $\stackrel{28}{=}$ We begin with the correlated transport regime $t_{f} / t_{b} \ll 1$ (cf. Fig. 1 in Ref. 28), where polaron motion becomes possible only through emission/absorption of bosons. This will be the regime of main interest to us when discussing the $2 \mathrm{D}$ problem.

In Fig. 3 we compare spectral weights $A(k, \omega)$ at $k=$ $0, \frac{\pi}{2}$ and $\pi$ for $\Omega / t_{b}=2, t_{f} / t_{b}=0.1$. The agreement between MA (thin line, shifted upwards) and ED (thick line) results is very satisfactory, especially for the features with larger weights. The agreement is of similar quality throughout the whole Brillouin zone (not shown). The 
most prominent features is the low-energy polaron band, which disperses very little (its bandwidth is comparable to the smallest energy scale in the problem, $t_{f}$ ), and has a $q p$ weight that varies little with $k$.

For a more detailed comparison, we focus on the polaron band, and plot its energy $E(k)$ vs $k$ in Fig. 4. As expected, for $t_{f}=0$ the dispersion corresponds to pure 2nd NN hopping $-2 t_{2} \cos (2 k a)$, where $t_{2}$ is dynamically generated through the 3 -site, 3-boson processes. For $t_{f} \neq 0$, an additional term $-2 t_{f}^{*} \cos (k a)$ with a renormalized transfer amplitude $t_{f}^{*}$ is also present. Figs. 4a, b show quite good agreement between MA and ED. As expected, the agreement is better for the larger $\Omega / t_{b}=2$ value, where the probability of more extended clouds is reduced. However, even for the smaller $\Omega / t_{b}=1$, MA captures the dispersion quite accurately: most of the difference to the ED results is an overall shift independent of $k$. This suggests that more extended clouds will not further renormalize the effective hopping integrals, only lower the overall polaron formation energy. This is reasonable, because longer range effective hopping terms are more complicated to generate and involve many more sites than the 3 -site, 3 -boson process responsible for $t_{2}$. It is therefore probably safe to conclude that MA accurately describes the polaron's dynamics in the $t_{f} / t_{b} \ll 1$ region, so long as $\Omega / t_{f}$ is not too small so that the spatial Green's functions decay exponentially reasonably fast.

As $\Omega / t_{b}$ is decreased we expect to move in the regime of "strong fluctuations".28 The low-energy part of the spectral weight in this regime is shown in Fig. [5] for $\Omega / t_{b}=0.5, t_{f} / t_{b}=0.04$. Because the cost of exciting bosons is reduced, we expect to see many more lowerenergy features than in Fig. 3, and this is indeed the case. MA shows reasonable agreement with ED at the lower energies, however, some of the higher-energy peaks are either missing, or displaced, or combined in a single feature. In order to properly describe these peaks,

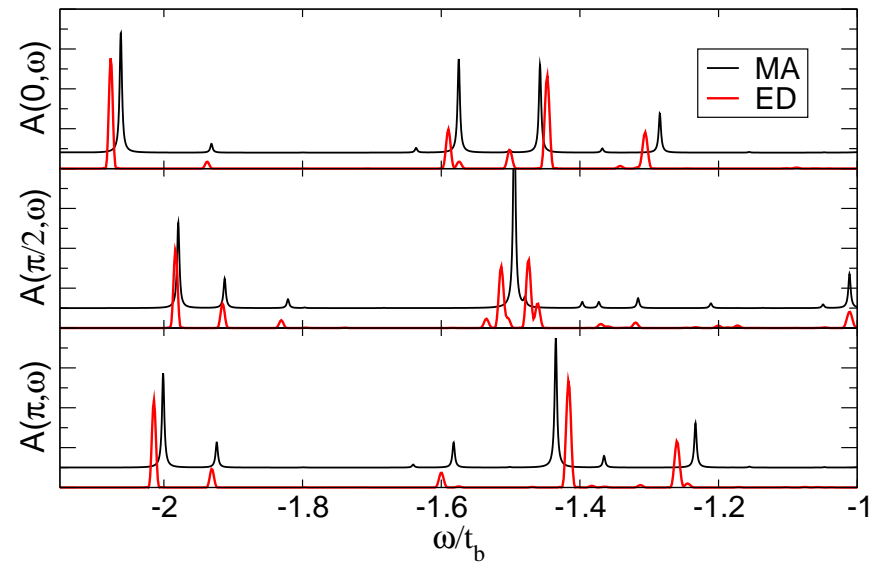

FIG. 5. (Color online) Spectral weights $A(k, \omega)$ for $k=0$ (top), $k=\frac{\pi}{2}$ (middle) and $k=\pi$ (bottom) from MA and ED. The MA results (black thin lines) are shifted upwards. The parameters are $\Omega / t_{b}=0.5, t_{f} / t_{b}=0.04, \eta / t_{b}=0.002$.

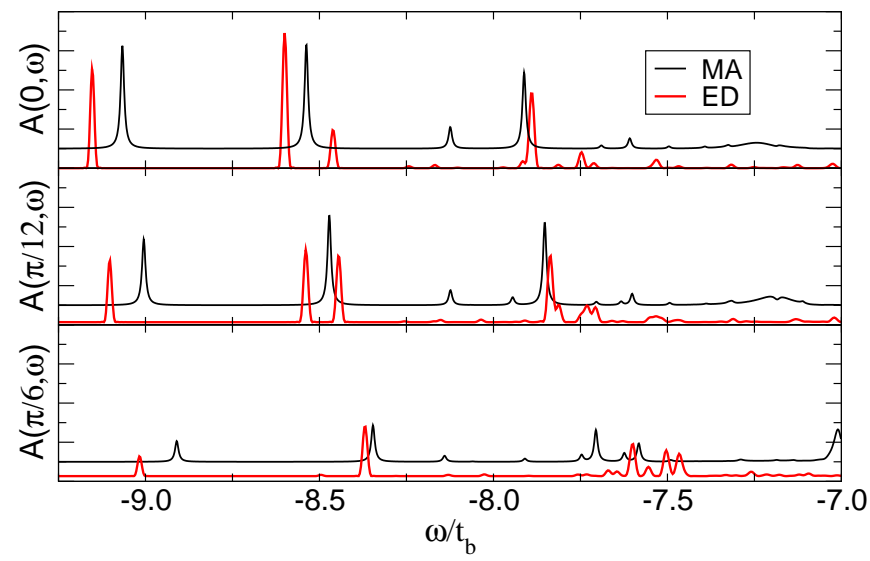

FIG. 6. (Color online) Spectral weights $A(k, \omega)$ for $k=0$ (top), $k=\frac{\pi}{12}$ (middle) and $k=\pi / 6$ (bottom) from MA and ED. Again the MA results are shifted upwards. The parameters are $\Omega / t_{b}=0.5, t_{f} / t_{b}=4, \eta / t_{b}=0.006$.

it is likely that one would need to go to the $\mathrm{MA}^{(1)}$ or higher level approximations, where bosons are allowed to exist far from the main polaron cloud (cf. Fig. 2(b)). Such states are essential in describing the polaron+one boson continuum starting at $E_{g s}+\Omega, 12,20$ and indeed, it is at these energies that the disagreements between MA and ED become more visible. If, however, the focus is on understanding the polaron band and if the polaron bandwidth is less than $\Omega$ (as is the case we discuss in $2 \mathrm{D}$, below), then inclusion of these states is not absolutely necessary: doing so will improve the quantitative agreement, of course, but will not change qualitatively the polaron dispersion.

Next we explore the "incoherent" or "diffuse" region of the parameter space, where $t_{b} / \Omega \gg 1$ while $t_{b} / t_{f} \ll$ 1.28 Since this implies $\Omega \ll t_{f}$, this is where the MA approximation is expected to be least accurate. In Fig. 6 we show comparisons of the spectral function $A(k, \omega)$ vs $\omega$ for small values of $k$. At $k=0$ (upper panel), ED shows two sharp peaks, associated with the polaron and the second bound state,,$\frac{12,20}{2}$ followed by the polaron+one boson continuum at $E_{g s}+\Omega$, and then more features at higher energies. MA finds the two peaks associated with the bound polaron states, shifted to slightly higher energies, but the continuum is absent since, as discussed, it is not included in the variational space at this MA level. As $k$ increases, the spectral weight (equal to the area under the peak) in the polaron band decreases extremely fast, similar to what happens for Holstein polarons at weak coupling, 11,20,40,41 and most of the weight shifts to roughly $\omega=\epsilon_{k}$. The lower panels of Fig. 6 illustrate this fast decrease in the polaron band spectral weight as $k$ increases. $\stackrel{41}{=}$ We also see that even though the polaron band predicted by MA is shifted upwards, this shift is again not strongly $k$ dependent. The $q p$ weight is also in good agreement with the ED results, so MA is still doing a reasonable job in predicting the $q p$ weight and effective mass. However, at larger $k$, the absence of the continuum 

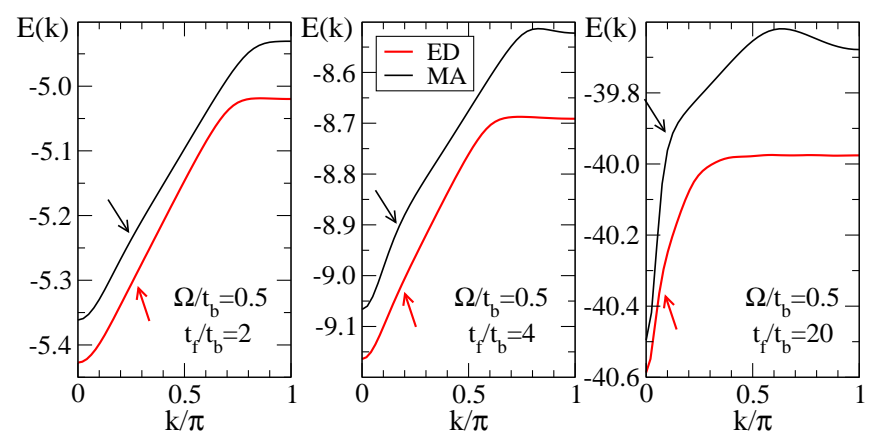

FIG. 7. (Color online) Polaron energy $E(k)$ in units of $t_{b}$ vs. $k$, for the parameters indicated. Dashed lines show MA results, full lines correspond to ED results. The arrows roughly mark a discontinuity in the slope of the dispersion.

within this level of MA approximation is expected to lead to an overestimate of the polaron bandwidth $\underline{\underline{19}}$

This is indeed the case, as shown in Fig. 7, where we compare the polaron dispersions $E(k)$ vs. $k$ for $\Omega=0.5 t_{b}$ and $t_{f} / t_{b}=2,4,20$, so that $\Omega / 2 t_{f}=0.125,0.0625$ respectively 0.0125 . The agreement at large $k$ worsens as $\Omega / t_{f} \rightarrow 0$. This is because in all these cases the bandwidth of the MA $E(k)$ exceeds $\Omega$, which is unphysical: the polaron band must always stay below the continuum. It follows that in this regime, the polaron + one boson continuum plays the key role in defining the polaron bandwidth. To describe it within MA, one needs to allow at least one boson to be far away from the polaron, i.e. to go to the $\mathrm{MA}^{(1)}$ level. The simpler MA ${ }^{(0)}$ level approximation used here is certainly untrustworthy at large $k$ in such cases. However, it still provides reasonably good description near $k=0$, as shown in Figs. 6 and 7 . For example, the ED polaron dispersion shows a "kink" at small $k$, whose origin we do currently understand. This feature is more pronounced as $\Omega / t_{f}$ decreases, and is indicated by the arrows in Fig. 7 MA also replicates it, albeit at somewhat different location. Below this value, the two dispersions seem to match quite well (up to an overall shift). We conclude that even in this least favorable regime and for this lowest level of MA, we can still use it to get reasonable estimates for the polaron effective mass and ground-state $q p$ weight. This is verified by the data presented in Table I] where we compare the effective masses (in dimensionless units),

$$
\frac{1}{m^{*}}=\left.\frac{1}{a^{2} t_{b}} \frac{\partial^{2} E(k)}{\partial k^{2}}\right|_{k=0},
$$

calculated with the two methods.

To summarize, these comparisons confirm that if the MA polaron bandwidth is less than $\Omega$ so that the continuum can be ignored, then this level of MA approximation suffices to describe $E(k)$ with good accuracy throughout the Brillouin zone. This is generically expected to hold so long as $\Omega / t_{f}$ is not too small, in other words for most of the parameter space. In the limit of small $\Omega / t_{f}$, the lowest MA approximation suffices for a reasonable de-

\begin{tabular}{|c|c|c|c|}
\hline$\Omega$ & $t_{f}$ & $m_{E D}^{*}$ & $m_{M A}^{*}$ \\
\hline \hline 1.0 & 0.00 & 17.71 & 18.12 \\
\hline 1.0 & 0.01 & 15.75 & 15.51 \\
\hline 2.0 & 0.00 & 129.28 & 129.98 \\
\hline 2.0 & 0.01 & 44.68 & 44.77 \\
\hline 0.5 & 2.00 & 1.54 & 1.39 \\
\hline 0.5 & 4.00 & 0.61 & 0.47 \\
\hline 0.5 & 20.00 & 0.052 & 0.041 \\
\hline
\end{tabular}

TABLE I. Comparison of effective masses as predicted by $\mathrm{ED}^{42}$ and MA, for several values of $\Omega$ and $t_{f}$ where $t_{b}=1$.

scription of GS properties, but one needs to go to higher MA levels and enlarge the variational space if one wants to have a good description of $E(k)$ in all the Brillouin zone. The same holds true if one wants an accurate description at energies above the polaron band. Because the MA approximations generically satisfy with good accuracy spectral weight sum rules,$\frac{19,20}{2}$ one expects even the lowest MA level to identify quite correctly where significant spectral weight appears in the spectrum. This is indeed the case, as shown in the comparisons provided here (regarding Fig. 7, one must remember that there is essentially no spectral weight in the $q p$ band once it gets close to the continuum). However, in order to capture finer details, one needs to work harder by suitably enlarging the variational space. In practice, this requires one to figure out the corresponding equivalent of Eqs. (14)-(26) and find an efficient way to solve them.

In the following, we focus on the polaron dispersion of the 2D Edwards model in the limit $t_{f} / t_{b} \rightarrow 0$ for a finite $\Omega / t_{b}$ ratio. In this case, the MA level introduced heresuitably generalized to $2 \mathrm{D}$-is sufficient for an accurate description of $E(k)$ in the entire Brillouin zone, therefore we do not need to go to a higher level.

\section{MA for the 2D square lattice}

To generate the MA equations - within the 3 -site cloud variational space - for a $2 \mathrm{D}$ square lattice, we follow the steps outlined in the previous section. The only complication is that now the 2 -site clouds can be aligned either along the $x$ or $y$ directions, so we need two types of 2 -site cloud generalized Green's functions,

$$
F_{n, m}^{(\boldsymbol{\eta})}(\mathbf{k}, \mathbf{q}, \omega)=\sum_{i} e^{i(\mathbf{k}-\mathbf{q}) \mathbf{R}_{i}}\left\langle 0\left|c_{\mathbf{k}} \hat{G}(\omega) c_{\mathbf{q}}^{\dagger} b_{i}^{\dagger m} b_{i+\boldsymbol{\eta}}^{\dagger}\right| 0\right\rangle
$$

with the associated

$$
\begin{array}{r}
f_{n, m}^{(\boldsymbol{\eta})}(\mathbf{k}, \boldsymbol{\delta}, \omega)=\frac{1}{N} \sum_{q} e^{i \mathbf{q} \delta a} F_{n, m}^{(\boldsymbol{\eta})}(\mathbf{k}, \mathbf{q}, \omega) \\
=\sum_{i} \frac{e^{i \mathbf{k} \mathbf{R}_{i}}}{\sqrt{N}}\langle 0| c_{\mathbf{k}} \hat{G}(\omega) c_{i-\boldsymbol{\delta}^{\dagger} b_{i}^{\dagger} b^{\dagger}{ }_{i+\boldsymbol{\eta}}^{n-m}|0\rangle,}
\end{array}
$$


where $\boldsymbol{\eta}=(1,0)=\mathbf{x}$ or $\boldsymbol{\eta}=(0,1)=\mathbf{y}$, each site index is two-dimensional, e.g. $i=\left(i_{x}, i_{y}\right)$, and we used the shorthand notation $i+\boldsymbol{\eta}=\left(i_{x}+1, i_{y}\right)$ if $\boldsymbol{\eta}=\mathbf{x}$ etc. As in $1 \mathrm{D}$, we ask that $1 \leq m \leq n-1$ to avoid overlap with the 1 -site cloud functions.

For the 3 -site clouds, we define:

$$
\begin{aligned}
& F_{n, m, p}^{\left(\boldsymbol{\eta}, \boldsymbol{\eta}^{\prime}\right)}(\mathbf{k}, \mathbf{q}, \omega)=\sum_{i} e^{i(\mathbf{k}-\mathbf{q}) \mathbf{R}_{i}}\left\langle 0\left|c_{\mathbf{k}} \hat{G}(\omega) c_{\mathbf{q}}^{\dagger}{b^{\dagger}}_{i-\boldsymbol{\eta}^{m}} b_{i}^{\dagger}{ }^{n-m-p} b_{i+\boldsymbol{\eta}^{\prime}}^{\dagger}\right| 0\right\rangle \\
& f_{n, m, p}^{\left(\boldsymbol{\eta}, \boldsymbol{\eta}^{\prime}\right)}(\mathbf{k}, \boldsymbol{\delta}, \omega)=\frac{1}{N} \sum_{q} e^{i \mathbf{q} \boldsymbol{\delta} a} F_{n, m, p}^{\left(\boldsymbol{\eta}, \boldsymbol{\eta}^{\prime}\right)}(\mathbf{k}, \mathbf{q}, \omega)=\sum_{i} \frac{e^{i \mathbf{k} \mathbf{R}_{i}}}{\sqrt{N}}\left\langle 0\left|c_{\mathbf{k}} \hat{G}(\omega) c_{i-\boldsymbol{\delta}}^{\dagger}{b^{\dagger}}_{i-\boldsymbol{\eta}}^{m} b_{i}^{\dagger}{ }^{n-m-p} b_{i+\boldsymbol{\eta}^{\prime}}^{\dagger}\right| 0\right\rangle .
\end{aligned}
$$

To describe collinear clouds we take $\boldsymbol{\eta}=\boldsymbol{\eta}^{\prime}$, and we again need only keep $\boldsymbol{\eta}=\mathbf{x}, \mathbf{y}$ for the two possible orientations. For non-collinear clouds we have 4 different distinct possibilities which we choose as $\boldsymbol{\eta}= \pm \mathbf{x}=( \pm 1,0)$ and $\boldsymbol{\eta}^{\prime}= \pm \mathbf{y}=(0, \pm 1)$, as sketched in Fig. 8. They are inequivalent except when $m+p=n$, so that there are bosons only on the opposite diagonal sites. Again, only $m \geq 1, p \geq 1$ are allowed.

The equations for the various $F$ and $f$ functions are generated just as in the $1 \mathrm{D}$ case, using the restriction that boson addition contributions cannot extend the boson cloud to more than 3 neighboring sites. This again leads to a set of equations for a finite number of $n$-boson functions $f$ which depend only on $n-1$ and $n+1$ boson $f$ functions. While straightforward to obtain, these equations are very lengthy (e.g. the equation for $f_{n}(\mathbf{k}, \boldsymbol{\delta}, \omega)$ has 64 contributions on the right-hand side), and we do not list all of them here. As an example, the relevant equations for the 3 -site cloud functions are listed below, using again the shorthand notations $f_{n, m, p}^{\left(\boldsymbol{\eta}, \boldsymbol{\eta}^{\prime}\right)}(\boldsymbol{\delta}) \equiv f_{n, m, p}^{\left(\boldsymbol{\eta}, \boldsymbol{\eta}^{\prime}\right)}(\mathbf{k}, \boldsymbol{\delta}, \omega)$ and $\omega_{n}=\omega-\Omega$ :

$$
\begin{aligned}
f_{n, m, p}^{\left(\boldsymbol{\eta}, \boldsymbol{\eta}^{\prime}\right)}(\boldsymbol{\delta})= & -m t_{b} \sum_{\boldsymbol{\delta}^{\prime}= \pm \mathbf{x}, \pm \mathbf{y}} g_{0}\left(\boldsymbol{\delta}+\boldsymbol{\delta}^{\prime}-\boldsymbol{\eta}, \omega_{n}\right) f_{n-1, m-1, p}^{\left(\boldsymbol{\eta}, \boldsymbol{\eta}^{\prime}\right)}(\boldsymbol{\eta})-(n-m-p) t_{b} \sum_{\boldsymbol{\delta}^{\prime}= \pm \mathbf{x}, \pm \mathbf{y}} g_{0}\left(\boldsymbol{\delta}+\boldsymbol{\delta}^{\prime}, \omega_{n}\right) f_{n-1, m, p}^{\left(\boldsymbol{\eta}, \boldsymbol{\eta}^{\prime}\right)}(0) \\
& -p t_{b} \sum_{\boldsymbol{\delta}^{\prime}= \pm \mathbf{x}, \pm \mathbf{y}} g_{0}\left(\boldsymbol{\delta}+\boldsymbol{\delta}^{\prime}+\boldsymbol{\eta}^{\prime}, \omega_{n}\right) f_{n-1, m, p-1}^{\left(\boldsymbol{\eta}, \boldsymbol{\eta}^{\prime}\right)}\left(-\boldsymbol{\eta}^{\prime}\right)-t_{b} g_{0}\left(\boldsymbol{\delta}+\boldsymbol{\eta}, \omega_{n}\right) \sum_{\boldsymbol{\delta}^{\prime}= \pm \mathbf{x}, \pm \mathbf{y}} f_{n+1, m+1, p}^{\left(\boldsymbol{\eta}, \boldsymbol{\eta}^{\prime}\right)}\left(\boldsymbol{\eta}+\boldsymbol{\delta}^{\prime}\right) \\
& -t_{b} g_{0}\left(\boldsymbol{\delta}, \omega_{n}\right) \sum_{\boldsymbol{\delta}^{\prime}= \pm \mathbf{x}, \pm \mathbf{y}} f_{n+1, m, p}^{\left(\boldsymbol{\eta}, \boldsymbol{\eta}^{\prime}\right)}\left(\boldsymbol{\delta}^{\prime}\right)-t_{b} g_{0}\left(\boldsymbol{\delta}-\boldsymbol{\eta}^{\prime}, \omega_{n}\right) \sum_{\boldsymbol{\delta}^{\prime}= \pm \mathbf{x}, \pm \mathbf{y}} f_{n+1, m, p+1}^{\left(\boldsymbol{\eta}, \boldsymbol{\eta}^{\prime}\right)}\left(\boldsymbol{\delta}^{\prime}-\boldsymbol{\eta}^{\prime}\right)
\end{aligned}
$$

An analysis of the terms occurring on the right-hand sides of these equations allows one to identify the minimum set of values $\boldsymbol{\delta}$ needed for the various $f$ functions. These are shown explicitly for a collinear and one noncollinear cloud in Fig. 9. There, the thick lines mark the position of the cloud, which is centered at $i$, while the dots show the locations of the fermion. The distances from the fermion to the site $i$ define the needed set of $\boldsymbol{\delta}$ values for these clouds. The other 3 -site clouds have sets related by appropriate symmetries. The sets for the 2 -site and 1-site clouds are found similarly.

Once this is done, the solution follows that used in 1D. All functions with a given $n$ are collected in a vector $V_{n}$. The equations of motions again reduce to matrix recurrence equations $V_{n}=\alpha_{n}(k, \omega) V_{n-1}+\beta_{n}(k, \omega) V_{n+1}$ which are solved in precisely the same way. Of course, the dimension of $V_{n}$ is now significantly increased, $\operatorname{dim}\left(V_{n}\right)=$ $31 n^{2}-15 n-3$, and therefore the various matrices
$A_{n}, \alpha_{n}, \beta_{n}$ needed are larger than in $1 \mathrm{D}$, however they are still very manageable. Most results shown below converged with relative errors less than $10^{-4}$ if we started from $A_{N}=0$ with $N=9$ or less, so that the largest vectors' dimension is below 2000. Moreover, their dimensions decrease fast as $n$ decreases, so the solution is still very efficient. In the plots shown below, a data point typically takes around a minute or less to generate.

We begin with a thorough analysis of the most interesting case, when $t_{f}=0$. In this case, only the 3 site, 3-boson terms already discussed will lead to dynamic generation of a polaron dispersion. We already know from the 1D case that we expect the generation of terms of the type $\sim-2 t_{3}\left[\cos \left(2 k_{x} a\right)+\cos \left(2 k_{y} a\right)\right]$ from the collinear clouds (on the $2 \mathrm{D}$ lattice, this corresponds to effective $3 \mathrm{rd} \mathrm{NN}$ hopping, hence $t_{3}$ ). However, because of the closed path (Trugman loops) processes that are now also possible, we also expect dynamic genera- 


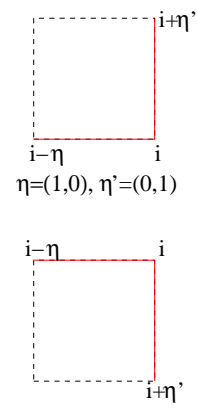

$\eta=(1,0), \eta^{\prime}=(0,-1)$
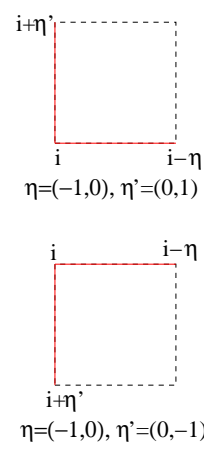

FIG. 8. (Color online) Sketch of our choice of $\boldsymbol{\eta}, \boldsymbol{\eta}^{\prime}$ indices for the $n$-boson non-collinear 3 -site boson clouds on the $2 \mathrm{D}$ square lattice. In all cases, $m \geq 1$ bosons are at site $i-\boldsymbol{\eta}$, $p \geq 1$ bosons are at site $i+\boldsymbol{\eta}^{\prime}$ and the remaining $n-m-p$ bosons are at site $i$.

tion of second $\mathrm{NN}$ hopping, leading to terms of the type $\sim-2 t_{2}\left[\cos \left(\left(k_{x}+k_{y}\right) a\right)+\cos \left(\left(k_{x}-k_{y}\right) a\right)\right]$. Altogether, then, in this case the polaron dispersion should be well described by

$$
\begin{aligned}
E(\mathbf{k})= & E_{P}-2 t_{2}\left[\cos \left(\left(k_{x}+k_{y}\right) a\right)+\cos \left(\left(k_{x}-k_{y}\right) a\right)-2\right] \\
& -2 t_{3}\left[\cos \left(2 k_{x} a\right)+\cos \left(2 k_{y} a\right)-2\right],
\end{aligned}
$$

where $E_{P}=E(\mathbf{k}=0)$ is the polaron ground state energy. From the 1D analysis, we expect that $t_{2}$ and $t_{3}$ should be quite accurately predicted by MA, while $E_{P}$ is only accurate at fairly high $\Omega$ and becomes systematically underestimated as $\Omega$ decreases.

In Fig. 10 we plot the polaron dispersion $E(\mathbf{k})$ and $q p$ weight $Z(\mathbf{k})$ along lines of high symmetry in the Brillouin zone. Interestingly, the two curves have similar profiles, however the $q p$ weight changes very little in real terms. This is somewhat reminiscent of Holstein polarons in the strong-coupling limit, which also have an almost constant $q p$ weight throughout the Brillouin zone. However, in that limit their $q p$ weight is exponentially small and the effective mass is exponentially large, whereas here the $q p$ weight is still considerable, as is the polaron bandwidth. This shows that very different physics gives rise to this behavior. Indeed, the small Holstein polaron has a small-size cloud with a large average number of bosons.
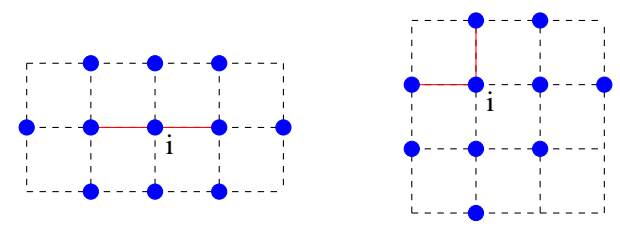

FIG. 9. (Color online) Pictorial description of the minimal sets of values $\boldsymbol{\delta}$ needed for a collinear (left) and non-collinear (right) 3 -site cloud generalized function $f_{n, m, p}(\boldsymbol{\delta})$. Red solid lines indicate the boson cloud centered at $i$, while the blue dots mark the possible locations of the fermion. The distances from the fermion to the site $i$ define the needed set of $\boldsymbol{\delta}$ values.
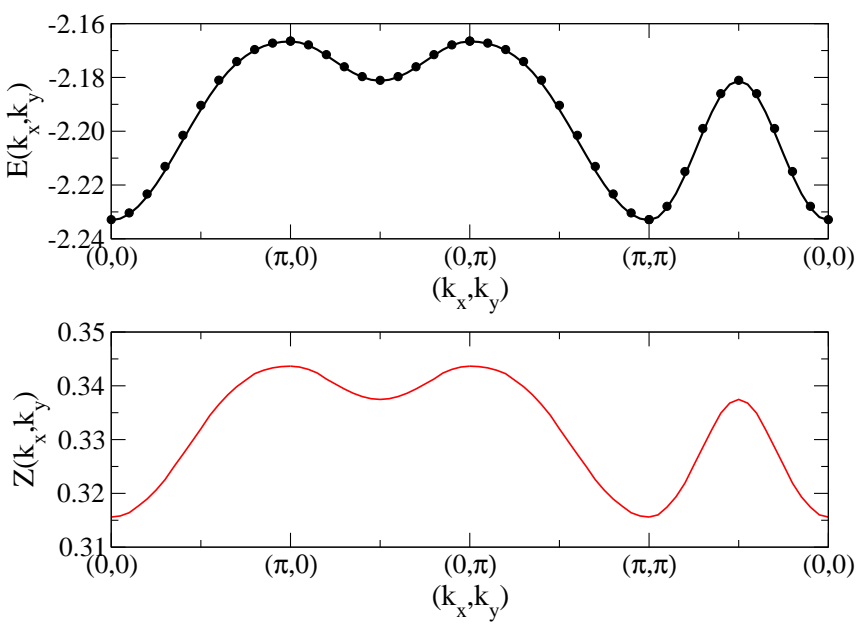

FIG. 10. (Color online) Polaron dispersion $E(\mathbf{k})$ (upper panel) and $q p$ weight $Z(\mathbf{k})$ (lower panel) along the highsymmetry directions in the Brillouin zone of the $2 \mathrm{D}$ square lattice, for $t_{f}=0, t_{b}=\Omega=1$. Lines show MA results, the symbols are fits to Eq. (41).

This explains its very large effective mass (due to vanishing overlap of the spatially small polaron cloud), the very small $q p$ weight (free fermion contribution to the wavefunction is very small), and its weak sensitivity to $\mathbf{k}$ (states that are nearly localized in real space are "extended" in k-space). In contrast, for the Edwards model polaron at $t_{f}=0$, all the dispersion is due to the existence of bosons through the boson-assisted hopping. As illustrated in Fig. 1, the free fermion state mixes with the various fermion+bosons states to give rise to the effective 2nd and 3rd NN hopping, so the fairly significant $q p$ weight throughout the Brillouin zone is not surprising. It is worth emphasizing again that the doubling of the Brillouin zone is due to the boson-modulated hopping. In fact, the resulting dispersion is somewhat reminiscent of that of a doped hole in a cuprate layer, although there the minimum is at $\left(\frac{\pi}{2 a}, \frac{\pi}{2 a}\right)$, which here is a saddle point.

The symbols in Fig. 10 are fits to Eq. (41). Specifically, we used the MA values for $E(0,0), E\left(\frac{\pi}{2}, \frac{\pi}{2}\right)$ and $E(0, \pi)$ in order to extract $E_{P}, t_{2}$ and $t_{3}$ from Eq. (41), and use these to generate the dispersion-fit in the entire Brillouin zone. The agreement is very reasonable, backing up our assumptions about polaronic physics in this regime. The only problem is that $t_{3} \ll t_{2}, E_{P}$, and as a result the level of confidence in extracting this parameter is not very high. For example, if we use $E\left(0, \frac{\pi}{2}\right)$ instead of $E\left(\frac{\pi}{2}, \frac{\pi}{2}\right)$ as the 3 rd point, the value of $t_{3}$ changes from 0.0023 to $0.0018\left(E_{P}, t_{2}\right.$ remain unchanged) but the agreement between the overall fit and $E(\mathbf{k})$ is visibly poorer.

In Fig. 11 we study the dependence of $t_{2}, t_{3}$ and $E_{P}$ on $\Omega / t_{b}$ when $t_{f}=0$. The full lines show converged MA results (except for very small $\Omega$, see below), whereas the symbols show the MA results with the restriction that we only allow clouds with up to 3 bosons $\left(A_{4}=0\right)$. As expected, at large $\Omega$ the agreement is very good: we know 


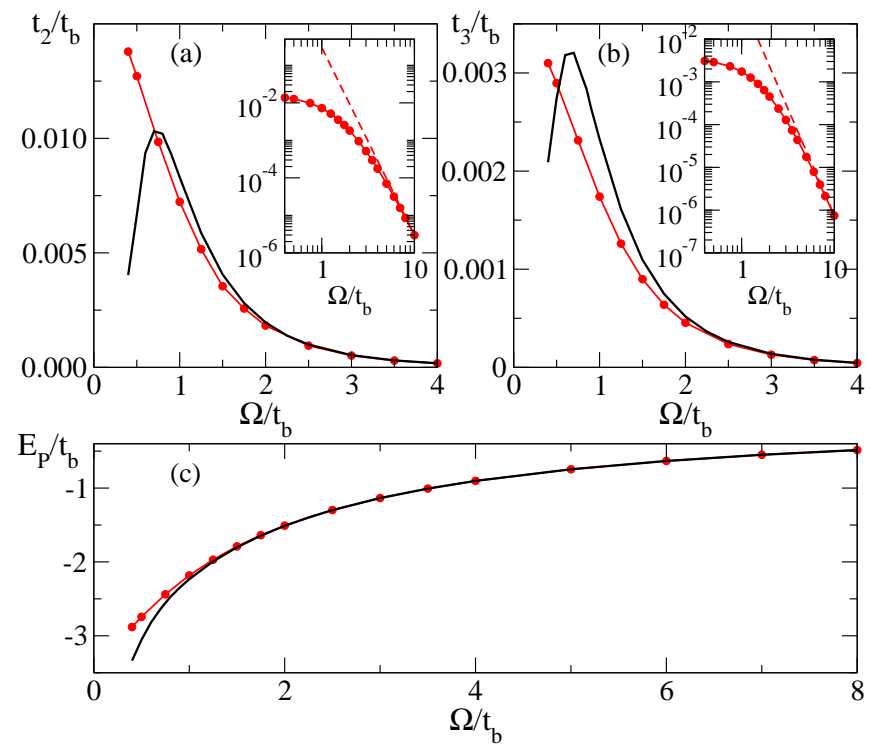

FIG. 11. (Color online) Effective $t_{2} / t_{b}$ in (a), $t_{3} / t_{b}$ in (b) and $E_{P} / t_{b}$ in (c), vs. $\Omega / t_{b}$ for $t_{f}=0$. The thick lines give fully converged MA results, the dots corresponds to the 3-boson solution. The insets show the 3 -boson results over a larger $\Omega / t$ range, and the dashed lines show $1 / x^{5}$ dependence. See text for more details.
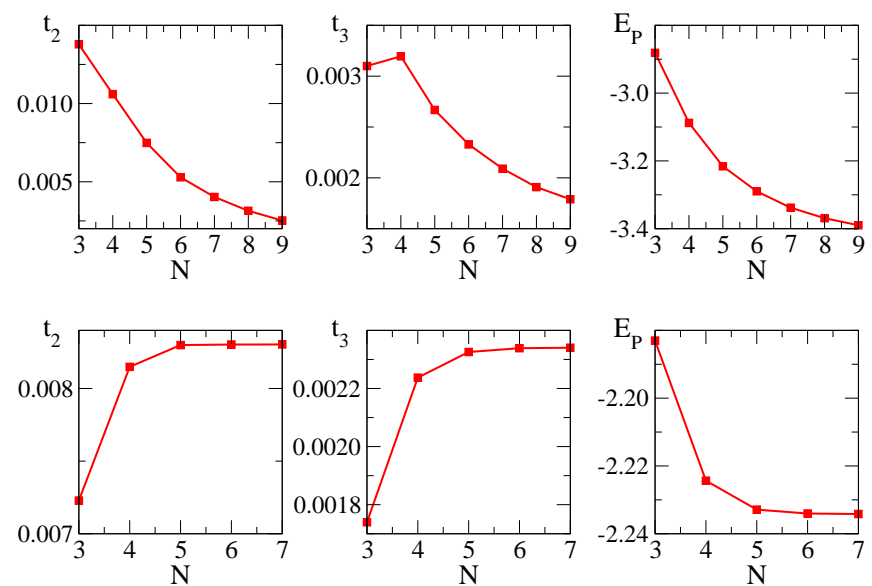

FIG. 12. Effective hopping integrals $t_{2}, t_{3}$ and polaron ground-state energy $E_{P}$ vs. the maximum number of allowed bosons $N$. The upper (lower) panels correspond to $\Omega / t_{b}=0.4$ $\left(\Omega / t_{b}=1\right)$. In both cases, $t_{f}=0$.

that we need clouds with at least 3 bosons to generate the effective hoppings, and because $\Omega$ is large, it is very unlikely to have larger clouds. This is further confirmed by the insets, which show that in the limit $t_{b} / \Omega \rightarrow 0$, both effective hoppings scale like $t_{b}^{6} / \Omega^{5}$, as expected for the 3-boson, 3-site processes from perturbation theory. The fits also confirm that in this limit, $t_{2} / t_{3}=4$. This is because there is constructive interference in going about the Trugman loops clockwise and anticlockwise to generate $t_{2}$, while $t_{3}$ can only be generated in a unique way.

As $\Omega$ decreases below roughly $2 t_{b}$, we see that $t_{2}, t_{3}$ be-
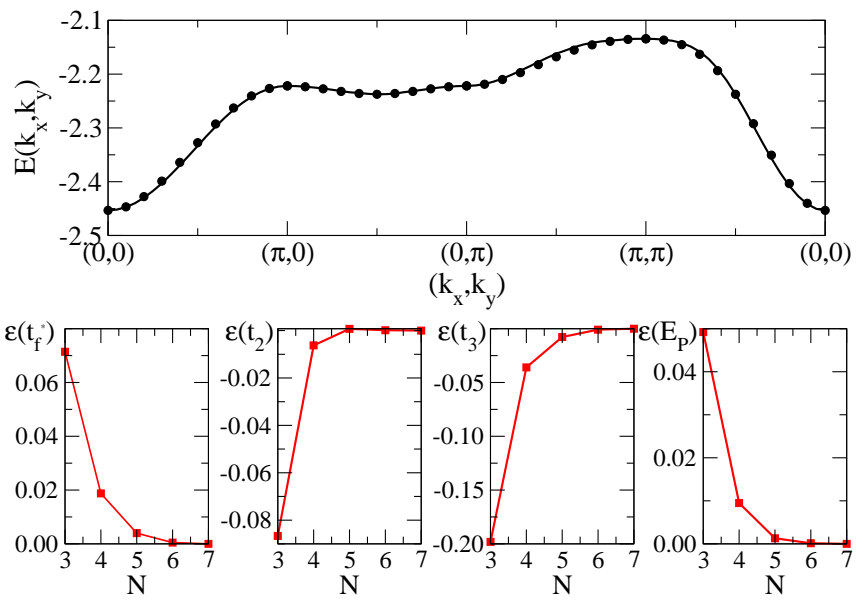

FIG. 13. Polaron dispersion $E(\mathbf{k})$ (upper panel) along highsymmetry cuts in the $2 \mathrm{D}$ square-lattice Brillouin zone, for $t_{f}=0.1, t_{b}=\Omega=1$. Lines show MA results, the symbols are fits to Eq. (42). The lower panels show the relative error $\varepsilon(x)=1-x(N) / x(\infty)$ in the effective hopping integrals, as well as the polaron ground-state energy, vs. the maximum number $N$ of allowed bosons.

come, at first, larger than the corresponding 3-boson values. Indeed, here we have to use larger clouds to achieve full convergence, and processes with more than 3 bosons will further increase the effective hoppings. Surprisingly, for $\Omega / t_{b}<0.7$ or so, $t_{2}$ and $t_{3}$ start to decrease fast. Here many-boson processes lead to a decrease of the effective hoppings from what the simple 3 -boson scenario would predict. Convergence of the various quantities in dependence on the maximum number $N$ of bosons allowed in the cloud is shown in Fig. 12 for $\Omega / t_{b}=0.4$ (upper panels) and for $\Omega / t_{b}=1$ (lower panels). The data indeed confirm that 4 and more boson terms have different effects on the effective hoppings for $\Omega<t_{b}$ and $\Omega>t_{b}$.

It is also clear that in the limit $\Omega / t_{b} \rightarrow 0$ our results are untrustworthy, because we ignore longer loops that also contribute to the effective hoppings in this regime. For example, just as the 6 -step Trugman loop on a $2 \times 2$ plaquette contributes to 2nd NN hopping, the Trugman loops on $2 \times 3$ plaquettes will contribute to both 2 nd and 3rd NN hopping (depending on how the fermion goes around the loop) and these contributions will supplement the values obtained from the processes included here. Its contributions scale asymptotically like $t_{b}^{11} / \Omega^{10}$ because they involve a 5-boson cloud and 11 hoppings to first create and then annihilate all of them. This is to be contrasted to $t_{b}^{6} / \Omega^{5}$ scaling for the contributions included in the 3 -site cloud MA. Clearly, once $\Omega \sim t_{b}$ we cannot ignore the contribution of these longer loops. This is why it is also pointless to go to larger $N$ to find the fully converged values for the $\Omega / t_{b}=0.4$ in Fig. 12. However, a good estimate of the crossover is difficult to obtain from such perturbation theory arguments, since the insets in Fig. 11 reveal that the asymptotic expressions are only valid at much larger $\Omega / t_{b}$ values. 

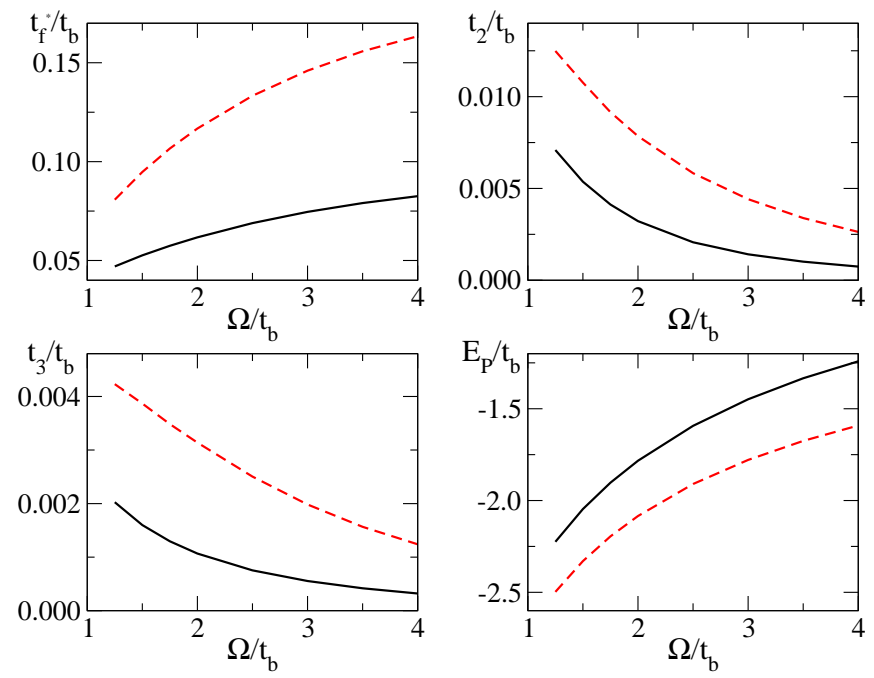

FIG. 14. (Color online) The effective hopping integrals and the polaron energy, in units of $t_{b}$, vs. $\Omega / t_{b}$, for $t_{f}=0.1 t_{b}$ (black solid line) and $t_{f}=0.2 t_{b}$ (red dashed line). The results are only shown for $\Omega$ values where 5 or more boson processes become irrelevant.

A better criterion is to take the value $\Omega / t_{b}$ above which convergence is achieved for a cutoff equal to or less than 6 , signaling that 5 or more boson processes are not contributing much to the polaron wavefunction, and therefore longer loops can be ignored safely. This definition is not going to produce a very sharp value since these contributions change gradually. From Fig. 12 we see that for $\Omega / t_{b}=1$, the change in going from $N=4$ to $N=5$ modifies various quantities by up to about $4 \%$, therefore this is likely already in the regime where longer loops are not playing an important role. Once 5-boson processes become important, we need to include in the variational calculation at least the $3 \times 2$ loops which will modify both $t_{2}$ and $t_{3}$. This is why the numbers shown in Fig. 111 are likely not valid for small $\Omega$. This is an example of how a MA approximation can signal its potential problems, but also how to fix them (here, extension to at least 5-site polaron clouds is needed at lower $\Omega$ ).

In any case, the boson-modulated hopping is responsible for dynamically generating a finite effective (dimensionless) mass $m^{*}=t_{b} /\left(t_{2}+2 t_{3}\right) \geq 14$ or so, even though the free particle has an infinite mass.

We next discuss the case of finite $t_{f}$, in the limit $t_{f}<\Omega$ and $\Omega / t_{b} \geq 1$ where the results of this approximation are expected to be valid. The main change in the polaron dispersion is that it will also acquire NN contributions, so now

$$
\begin{aligned}
E(\mathbf{k})= & E_{P}-2 t_{f}^{*}\left[\cos \left(k_{x} a\right)+\cos \left(k_{y} a\right)-2\right] \\
& -2 t_{2}\left[\cos \left(\left(k_{x}+k_{y}\right) a\right)+\cos \left(\left(k_{x}-k_{y}\right) a\right)-2\right] \\
& -2 t_{3}\left[\cos \left(2 k_{x} a\right)+\cos \left(2 k_{y} a\right)-2\right],
\end{aligned}
$$

where $t_{f}^{*}$ is renormalized due to polaron cloud overlap.

The polaron dispersion is shown in Fig. 13 for $t_{f}=$ $0.1 t_{b}, \Omega=t_{b}$. A comparison with Fig. 10 reveals that the small $t_{f}$ has a significant effect on $E(\mathbf{k})$, especially near the edges of the Brillouin zone. The symbols show fits to Eq. (42). Here, the values for $E_{P}, t_{f}^{*}, t_{2}$ and $t_{3}$ were extracted from the energies at the special points $(0,0),\left(\frac{\pi}{2}, \frac{\pi}{2}\right),(0, \pi)$, and $(\pi, \pi)$. Using these parameters in Eq. (42) leads to good agreement with the MA results (thick lines). We find that $t_{f}^{*} / t_{b}=0.040, t_{2} / t_{b}=$ $0.0090, t_{3} / t_{b}=0.0026$. Interestingly, while $t_{f}^{*}<t_{f}$, as expected in polaronic physics, we see that in the presence of a finite $t_{f}$, both $t_{2}$ and $t_{3}$ are larger than when $t_{f}=0$. This is because the effective hopping integrals generated by the 3-boson processes discussed so far are here supplemented by the usual longer-range polaron hopping known to occur in the intermediate-to-strong electron-phonon coupling limit. 40,41 Just as for $t_{f}=0$, the $q p$ weight changes little throughout the Brillouin zone; it is close to 0.3 everywhere. The lower panels in Fig. 13 show the convergence of the various effective hopping amplitudes and of the ground-state energy as the maximum number of bosons $N$ increases. Specifically, we plotted the relative errors which reveal that 5 or more boson processes contribute less than $4 \%$ to the various quantities.

The dependence of $t_{f}^{*}, t_{2}, t_{3}$ and $E_{P}$ on $\Omega / t$ is shown in Fig. 14 for a two values of $t_{f}$. The data is only displayed over the range where 5 -boson processes contribute less than $1 \%$ to the various parameters, so that longer Trugman loops can be safely ignored. As expected, $t_{f}^{*}$ increases towards $t_{f}$ as $\Omega$ increases, because this leads to fewer bosons in the polaronic cloud, i.e. less "dressing" of the quasiparticle. On the other hand, $t_{2}$ and $t_{3}$ decrease with increasing $\Omega$, as this makes the intermediary manyboson states less likely. As already discussed, their values increase with increasing $t_{f}$, for a fixed value of $\Omega$. Finally, we note that $E_{P}$ is well below the free particle continuum starting at $-4 t_{f}$. This, together with the fact that longer loops are irrelevant, guarantees that the approximation must be quantitatively accurate in this regime.

\section{SUMMARY AND DISCUSSIONS}

The main goal of this work is to demonstrate how the MA approximations can be generalized to study polaron formation in models with boson-affected fermion hopping. Unlike for simpler local fermion-boson couplings as in the Holstein polaron model, where the strong-coupling Lang-Firsov solution is known and can guide the choice for the maximum extension of the polaron cloud, here this solution is not available. As illustrated for the Edwards fermion-boson model, one now needs to use physical intuition to make a reasonable choice. Of course, one can always systematically increase the variational space and check that the initial guess was indeed reasonable.

Because we are interested primarily in the low-energy polaron physics, we used the $\mathrm{MA}^{(0)}$ level of approximation which describes only the polaron cloud and does not allow for far-flung bosonic excitations. Then, the only free "parameter" is the spatial size of the polaronic cloud. 
Simple arguments regarding the processes illustrated in Fig. 1 show that at least 3 -site clouds need to be allowed, and therefore we built the approximation for this case. Careful consideration of the terms ignored gives us intuition about when the approximation is expected to be accurate, and when and in which way it becomes problematic. In 1D, this was indeed verified successfully against available exact numerical results.

We then extended the calculation to $2 \mathrm{D}$, where no results were available for this model until now, and demonstrated that the closed Trugman loops play the key role in determining the effective mass of the quasiparticle in the limit $t_{f}=0$. In this regime, the results for the 3 -site MA calculation are trustworthy for $\Omega>t_{b}$; for smaller $\Omega$ values, one needs to increase the allowed size of the polaron cloud since longer Trugman loops are also becoming important. We emphasize that the MA approximation is not wrong in the low $\Omega$ limit; what failed is our guess about the relevant size of the polaron cloud. If this is increased from 3 to more sites, the approximation will become accurate again.

The important role played by Trugman loops raises a very important question regarding the motion of a particle through an AFM background (which, as discussed, the Edwards fermion-boson model partly mimics). For $t-J$ models, it has been argued that the interaction of the hole with spin-waves is well described within the selfconsistent Born approximation. This approximation includes only non-crossing diagrams, i.e. processes where the bosons are absorbed in inverse order to the one in which they have been emitted by the particle. This is because it is generally expected that the particle needs to retrace its path to "heal" the string of defects it created when it reshuffled the spins. For a Neél AFM, the self-consistent Born approximation therefore predicts an infinitely heavy quasiparticle. In the presence of spinfluctuations, the magnons can disperse and this gives rise to a finite quasiparticle mass.

What is shown here is that the quasiparticle can acquire a finite spin mass even in the absence of spin fluctuations, by going (almost) twice around closed loops, first creating a string of defects and then healing it. Note that in diagrammatic terms, this would correspond to maximally crossed diagrams, since here the bosons are absorbed in the same order in which they were emitted. Such processes are obviously not included in the self-consistent Born approximation.

Of course, one might argue that spin fluctuations are relevant for holes in cuprates, in other words $t_{f}$ may be considerable and therefore dominate $E(k)$, as we found it to be the case for the Edwards model for $t_{f}>0$. In other words, that the Trugman loops' contributions, although finite, may be quantitatively insignificant. However, the interesting thing is that these closed loop processes give rise to the only contributions in $E(k)$ which are consistent with the doubling of the unit cell. ARPES on the parent insulators, i.e. for a single quasiparticle introduced in a $\mathrm{CuO}_{2}$ layer, clearly exhibits this doubling of the Brillouin zone $\stackrel{29}{=}$ This suggests that maybe this problem should be revisited using MA-type approximations.

\section{ACKNOWLEDGMENTS}

The authors would like to thank A. Alvermann and D. M. Edwards for valuable discussions. This work was supported by NSERC and CIfAR (MB), and by Deutsche Forschungsgemeinschaft through SFB 652 (HF).
1 H. Fröhlich, Adv. Phys. 3, 325 (1954).

2 Y. A. Firsov, Polarons (Izd. Nauka, Moscow, 1975).

3 C. L. Kane, P. A. Lee, and N. Read, Phys. Rev. B 39, 6880 (1989).

4 G. Martinez and P. Horsch, Phys. Rev. B 44, 317 (1991).

5 K. Wohlfeld, A. M. Oleś, and P. Horsch, Phys. Rev. B 79, 224433 (2009).

${ }_{6}$ M. Berciu, Physics 2, 55 (2009).

7 L. D. Landau, Phys. Z. Sowjetunion 3, 664 (1933).

${ }^{8}$ G. Iadonisi, J. Ranninger, and G. D. Filipis, eds., Polarons in bulk materials and systems with reduced dimensionality, vol. 161 of International School of Physics Enrico Fermi (IOS Press, Amsterdam, 2006).

9 A. S. Alexandrov, ed., Polarons in Advanced Materials, vol. 103 of Springer Series in Material Sciences (Springer, Dordrecht, 2007).

10 E. Jeckelmann and H. Fehske, Rivista del Nuovo Cimento 30, 259 (2007).

11 H. Fehske and S. A. Trugman, in Polarons in Advanced Materials, edited by A. S. Alexandrov (Canopus/Springer Publishing, Dordrecht, 2007), vol. 103 of Springer Series in Material Sciences, pp. 393-461.
12 J. Bonca, S. A. Trugman, and I. Batistic, Phys. Rev. B 60, 1633 (1999).

13 G. De Filippis, V. Cataudella, V. Marigliano Ramaglia, and C. A. Perroni, Phys. Rev. B 72, 014307 (2005).

14 P. E. Kornilovitch, Phys. Rev. Lett. 81, 5382 (1998).

15 M. Hohenadler, H. G. Evertz, and W. von der Linden, Phys. Rev. B 69, 024301 (2004).

16 N. V. Prokof'ev and B. V. Svistunov, Phys. Rev. Lett. 81, 2514 (1998).

17 T. Holstein, Ann. Phys. (N.Y.) 8, 325 (1959).

18 T. Holstein, Ann. Phys. (N.Y.) 8, 343 (1959).

19 M. Berciu, Phys. Rev. Lett. 97, 036402 (2006); G. L. Goodvin, M. Berciu, and G. A. Sawatzky, Phys. Rev. B 74, 245104 (2006).

20 M. Berciu, Phys. Rev. Lett. 98, 209702 (2007); M. Berciu and G. L. Goodvin, Phys. Rev. B 76, 165109 (2007).

21 G. L. Goodvin and M. Berciu, Phys. Rev. B 78, 235120 (2008).

22 L. Covaci and M. Berciu, Europhys. Lett. 80, 67001 (2007).

23 L. Covaci and M. Berciu, Phys. Rev. Lett. 100, 256405 (2008). 
${ }^{24}$ L. Covaci and M. Berciu, Phys. Rev. Lett. 102, 186403 (2009).

25 M. Berciu, A. S. Mishchenko and N. Nagaosa, Europhys. Lett. 8937007 (2010).

26 G. L. Goodvin, L. Covaci and M. Berciu, Phys. Rev. Lett. 103, 176402 (2009).

27 D. M. Edwards, Physica B 378-380, 133 (2006).

28 A. Alvermann, D. M. Edwards, and H. Fehske, Phys. Rev. Lett. 98, 056602 (2007).

29 A. Damascelli, Z. Hussain, and Z.-X. Shen, Rev. Mod. Phys. 75, 473 (2003).

30 S. A. Trugman, Phys. Rev. B 37, 1597 (1988).

31 O. S. Barisic, Phys. Rev. Lett. 98, 209701 (2007).

32 M. Berciu, Can. J. Phys 86, 523 (2008).

33 A. Alvermann, D. M. Edwards, and H. Fehske, J. Phys. Conf. Ser. 220, 012023 (2010).
${ }^{34}$ H. Fehske, A. Alvermann, and G. Wellein, in High Performance Computing in Science and Engineering, Garching/Munich 200\%, edited by S. Wagner, M. Steinmetz, A. Bode, and M. Brehm (Springer-Verlag, Berlin, 2009), pp. 649-668.

35 W. P. Su, J. R. Schrieffer, and A. J. Heeger, Phys. Rev. Lett. 42, 1698 (1979).

36 E. N. Economou, "Green's functions in quantum physics", Springer-Verlag, Berlin 1983.

37 G. Wellein and H. Fehske, Phys. Rev. B 58, 6208 (1998).

38 A. Alvermann, H. Fehske, and S. A. Trugman, Phys. Rev. B 81, 165113 (2010).

39 I. G. Lang and Y. A. Firsov, Zh. Eksp. Teor. Fiz. 43, 1843 (1962), [Sov. Phys. JETP 16, 1301 (1963)].

${ }^{40}$ W. Stephan, Phys. Rev. B 54, 8981 (1996).

41 G. Wellein and H. Fehske, Phys. Rev. B 56, 4513 (1997).

42 A. Alvermann, private communication (2010). 